\title{
Fabrication and characterization of a rapid prototyped tissue engineering scaffold with embedded multicomponent matrix for controlled drug release
}

\author{
This article was published in the following Dove Press journal: \\ International Journal of Nanomedicine \\ 2 August 2012 \\ Number of times this article has been viewed
}

\author{
Muwan Chen 1,2 \\ Dang QS Le I,2 $^{1,2}$ \\ San Hein ${ }^{2}$ \\ Pengcheng $\mathrm{Li}^{1}$ \\ Jens $V$ Nygaard ${ }^{2}$ \\ Moustapha Kassem ${ }^{3}$ \\ Jørgen Kjems ${ }^{2}$ \\ Flemming Besenbacher ${ }^{2}$ \\ Cody Bünger' \\ 'Orthopaedic Research Lab, Aarhus \\ University Hospital, Aarhus C, \\ Denmark; ${ }^{2}$ Interdisciplinary \\ Nanoscience Center (iNANO), \\ Aarhus University, Aarhus C, \\ Denmark; ${ }^{3}$ Department of \\ Endocrinology and Metabolism, \\ Odense University Hospital, \\ Odense C, Denmark
}

\begin{abstract}
Bone tissue engineering implants with sustained local drug delivery provide an opportunity for better postoperative care for bone tumor patients because these implants offer sustained drug release at the tumor site and reduce systemic side effects. A rapid prototyped macroporous polycaprolactone scaffold was embedded with a porous matrix composed of chitosan, nanoclay, and $\beta$-tricalcium phosphate by freeze-drying. This composite scaffold was evaluated on its ability to deliver an anthracycline antibiotic and to promote formation of mineralized matrix in vitro. Scanning electronic microscopy, confocal imaging, and DNA quantification confirmed that immortalized human bone marrow-derived mesenchymal stem cells (hMSC-TERT) cultured in the scaffold showed high cell viability and growth, and good cell infiltration to the pores of the scaffold. Alkaline phosphatase activity and osteocalcin staining showed that the scaffold was osteoinductive. The drug-release kinetics was investigated by loading doxorubicin into the scaffold. The scaffolds comprising nanoclay released up to $45 \%$ of the drug for up to 2 months, while the scaffold without nanoclay released $95 \%$ of the drug within 4 days. Therefore, this scaffold can fulfill the requirements for both bone tissue engineering and local sustained release of an anticancer drug in vitro. These results suggest that the scaffold can be used clinically in reconstructive surgery after bone tumor resection. Moreover, by changing the composition and amount of individual components, the scaffold can find application in other tissue engineering areas that need local sustained release of drug.
\end{abstract}

Keywords: nanoclay, chitosan, scaffold, tissue engineering, drug delivery system

\section{Introduction}

Repair of bone defects caused either by traumatic accidents or tumor resection poses major health care challenges to biomedical science and technology. ${ }^{1-3}$ Bone tissue engineering scaffolds have advanced greatly over the last decade and show very promising potential as bone graft substitutes. ${ }^{4,5}$ Scaffolds are generally composed of polymers and other materials which have been used in drug delivery systems for decades. The combined efforts of medical practitioners and material scientists enable fabrication of scaffolds with additional drug delivery features to which clinically important functionalities are added. To facilitate bone tissue formation, bioactive molecules like growth factors are incorporated into the scaffold. ${ }^{6,7}$ To prevent bacterial infection and biofilm, scaffolds have been designed to release antibiotics. ${ }^{8}$ To prevent cancer recurrence, chemotherapeutic drugs have been incorporated into the scaffold. ${ }^{9,10}$ There is an increasing interest in fabrication of drug-eluting bone tissue engineering 
scaffolds because these scaffolds provide an approach that conventional medical practice does not offer. ${ }^{11-13}$

One such drug-eluting scaffold is a ceramic scaffold which is osteoconductive and able to carry drugs. However, it is difficult to tune drug release profiles into desired levels. ${ }^{14}$ Biodegradable polymeric materials such as polycaprolactone (PCL) $)^{15}$ and poly (lactic-co-glycolic acid) (PLGA) ${ }^{16}$ have been investigated for bone tissue engineering and local drug delivery. There are several techniques in fabrication PCL scaffolds such as freeze drying, salt leaching and rapid prototyping. Among them, rapid prototyping or 3D printing are popular because it is possible to control the pore sizes and shapes of the scaffold to different degrees of strength and biodegradability. ${ }^{17}$ However, the exclusive use of PCL and PLGA scaffolds has shown limited success because of their hydrophobicity, difficulty to control drug release, and inferior osteoconductivity. Thus, an optimal combination of biomaterials is essential in the fabrication of tunable drug-eluting bone scaffolds with proper mechanical strength.

Nanoclay (sodium montmorillonite) are layered silicates, cationic exchangers, and possess negatively charged surfaces with specific surface areas up to $750 \mathrm{~m}^{2} / \mathrm{g} \cdot{ }^{18}$ Cationic drug or polymer molecules can intercalate and exfoliate the clay particles to provide a stable aqueous suspension and improve aqueous solubility of drugs. The cation exchange capacity of the clay, the charge of the drug, and $\mathrm{pH}$ of the medium determine the drug-release kinetics. Additional clay-drug interaction mechanisms, including hydrophobic, hydrogen bonding, ligand exchange, and water bridging may also be present. These properties have encouraged the use of clays for controlled release of drugs. ${ }^{19,20}$ Chitosan/clay nanocomposites are also potential sustained drug-release carriers. $^{21-23}$

Both chitosan and calcium phosphate compounds, eg, hydroxyapatite or $\beta$-tricalcium phosphate, are widely used in bone tissue engineering because of their osteogenic properties. In addition, studies have shown that incorporation of clay with chitosan and hydroxyapaptite improves both mechanical and osteogenic scaffold properties. ${ }^{24,25}$ However, the strength of the composite scaffold made from the combination of clay, $\beta$-tricalcium phosphate, and chitosan is insufficient to implant in defects of a high-loading tissue such as bone.

Therefore, the mechanically stable and biodegradable rapid prototyped macroporous PCL scaffold was used to host an osteoconductive and drug-eluting porous matrix. The chitosan/ $\beta$-tricalcium phosphate ( $\beta$-TCP) composite was embedded into the host scaffold to improve osteoconductivity of the scaffold. The drug-loaded sodium montmorillonite clay was further incorporated to the chitosan/ $\beta$-TCP matrix, providing a tunable drug-release system to the scaffold.

We used doxorubicin as a model drug because it is a widely used anthracycline antibiotic with a broad-spectrum antitumor activity to treat several types of malignancies, ${ }^{26-28}$ especially for soft tissue and bone sarcoma. ${ }^{29}$ However, due to its cumulative-dose limit and myocardial toxicity, treatment with doxorubicin is limited. ${ }^{30,31}$ Therefore, a sustained local drug delivery system could overcome these drawbacks.

We have designed and tested a biocompatible, biodegradable, and bioresorbable scaffold, capable of sustained drug release for a therapeutic strategy. The drug-loaded chitosan/ nanoclay/ $\beta$-TCP composite is housed in a rapid prototyped polycaprolactone scaffold for this purpose. We evaluated this composite scaffold in vitro in terms of its bone graft substitute potential with hMSC and its capacity for sustained release of doxorubicin.

\section{Materials and methods}

The nanoclay was Cloisite $\mathrm{Na}^{+}$, Lot: 07F28GDX-008 (Southern Clay Products, Inc, Moosburg, Germany). The chitosan was Chitopharm M with 75\%-85\% degree of deacetylation (Cognis, Florham Park, NJ). Polycaprolactone (MW =50 kDa) was from Perstorp (Cheshire, UK). The $\beta$-TCP nanocrystals were Lot: TCPCH01 (Berkeley Advanced Biomaterials, Inc, Berkeley, CA). Doxorubicin hydrochloride (DOX) was from Sigma-Aldrich (St Louis, MO).

\section{Scaffold fabrication PCL-base scaffold manufacture}

Scaffolds were made from PCL by means of fused deposition modeling with a BioScaffolder (SYS+ENG GmbH, Ilmenau, Germany). Using a biopsy punch (Acuderm, Fort Lauderdale, FL), cylindrical scaffolds with a diameter of $10 \mathrm{~mm}$ were punched out from $5 \mathrm{~mm}$-thick porous PCL mats. To increase surface hydrophilicity and thus improve cell attachment, the scaffolds were etched in $5 \mathrm{~mol} / \mathrm{L}$ sodium hydroxide for 3 hours, and then in $70 \%$ ethanol for sterilization. The scaffolds were rinsed in sterile water multiple times and dried.

\section{Clay modification}

Our pilot study showed that the clay-DOX carrier released less than $10 \%$ in 1 month. Thus we modified the clay with chitosan as described by Yuan et $\mathrm{al}^{23}$ and in the remainder of this paper, "clay" denotes this modified clay.

Clay was added into $0.2 \%(\mathrm{w} / \mathrm{v})$ chitosan solution prepared in $1.0 \%(\mathrm{v} / \mathrm{v})$ acetic acid. The weight ratio of chitosan to clay was 10:1. After stirring for 4 hours at $\sim 500 \mathrm{rpm}$, the colloidal 
suspension was centrifuged and washed three times with $1.0 \%$ (v/v) acetic acid in order to remove free chitosan. Finally, after dispersing the modified clay nanoparticles pellet in $1.0 \%(\mathrm{v} / \mathrm{v})$ acetic acid, it was ready for scaffold fabrication.

\section{Clay/DOX carrier}

The modified clay was dispersed in DOX solution for 12 hours and in vortex for 2 hours. Then the solution was centrifuged at $15,000 \mathrm{~g}$ for 10 minutes and the supernatant was collected. DOX was encapsulated into the clay nanoparticles and designated as clay/DOX carrier.

\section{Preparation of composite scaffolds}

$\beta$-TCP nanoparticles were dispersed in $1 \%(\mathrm{w} / \mathrm{v})$ chitosan solution prepared in $1 \%(\mathrm{v} / \mathrm{v})$ acetic acid. The weight ratio of $\beta$-TCP to chitosan was 1:20. The chitosan/ $\beta$-TCP solution was stirred at room temperature and then divided into four groups: A, B, C, and D, our testing groups for drug delivery (Figure 1).

Modified clay was added to Group A solution and used as a blank scaffold for the bone tissue engineering. DOX was added to Group B solution and used as a control group for the drug delivery. Both modified clay and DOX were added to Group C solution. The clay/DOX carrier was added to Group D solution. Each PCL scaffold (thickness $5 \mathrm{~mm}$ and diameter $10 \mathrm{~mm}$ ) was immersed in $500 \mu \mathrm{L}$ of each solution and was frozen at $-20^{\circ} \mathrm{C}$ for 24 hours. Subsequently, lyophilization was done at $-20^{\circ} \mathrm{C}$ at $40 \mathrm{mTorr}$ for 48 hours with a Dura-Stop/Dura-Dry freeze dryer system (FTS Systems; SP Scientific, Warminster, PA). Next, the scaffolds were neutralized in $0.4 \mathrm{M} \mathrm{NaOH}$ in $70 \%$ ethanol solution for 15 minutes initially and then in $70 \%$ ethanol for 3 hours for sterilization treatment. The scaffolds were

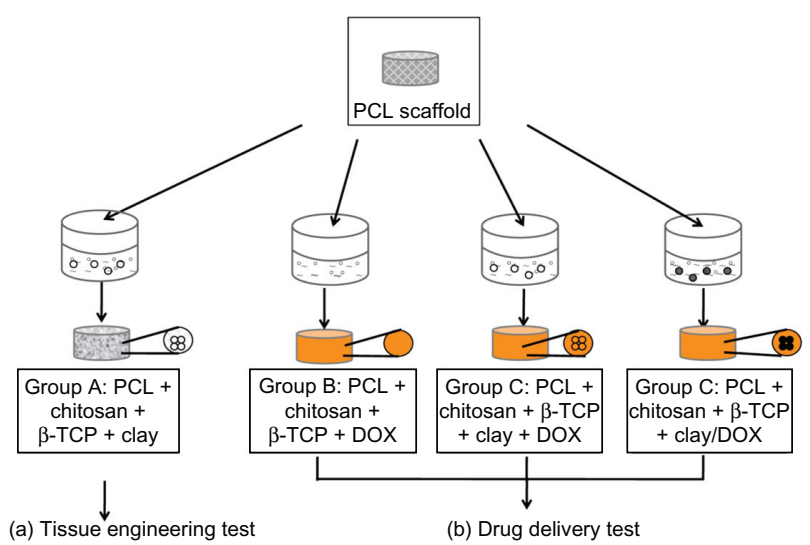

Figure I Schematic of composite scaffold preparation and groups.

Abbreviations: $\beta$-TCP, $\beta$-tricalcium phosphate; DOX, doxorubicin hydrochloride; $\mathrm{PCL}$, polycaprolactone.
Table I Scaffold composition in different groups

\begin{tabular}{lllll}
\hline Scaffold composition & A & B & C & D \\
\hline I\% chitosan $(\mu \mathrm{L})$ & 500 & 500 & 500 & 500 \\
$\beta-T C P(\mathrm{mg})$ & 0.25 & 0.25 & 0.25 & 0.25 \\
Clay $(\mathrm{mg})$ & 0.45 & - & 0.45 & - \\
DOX $(\mathrm{mg})$ & - & 0.1 & 0.1 & - \\
Clay/DOX $(\mathrm{mg})$ & - & - & - & $0.45 / 0.1$ \\
\hline
\end{tabular}

Abbreviations: $\beta$-TCP, $\beta$-tricalcium phosphate; DOX, doxorubicin hydrochloride.

rinsed in phosphate-buffered saline (PBS) multiple times and freeze-dried. The combinations of each scaffold are shown in Table 1.

\section{Drug-release profile test}

The release profile of DOX from the scaffold was determined by incubating a piece of scaffold in $1.0 \mathrm{~mL}$ of sterile PBS $(\mathrm{pH}=7.4)$ at $37^{\circ} \mathrm{C}(\mathrm{n}=4)$ in a sterile incubator for different time intervals. Scaffolds were placed in a 48 -well plate (one scaffold/well) and the lid was closed tightly. At each time point, $1 \mathrm{~mL}$ of solution was collected and replaced with $1 \mathrm{~mL}$ of fresh PBS. The fluorescence intensity of DOX in the buffer solution was quantified with a Victor 1420 multilabel counter (Wallac, Waltham, MA) with excitation at $405 \mathrm{~nm}$ and emission at $615 \mathrm{~nm}$. The concentrations of DOX released in the solutions were calculated according to the calibration curve of DOX in PBS and the cumulative release rates were calculated afterwards.

\section{Seeding hMSC-TERT cells to scaffold}

A telomerase reverse transcriptase gene-transduced cell population, hMSC-TERT cells, was used in this study. These cells maintain the functional characteristics of primary MSCs and have the capability to differentiate into certain mesodermal cell types (osteoblasts, chondrocytes, and adipocytes) in the presence of specific stimuli. ${ }^{32}$ Cells from population doubling (PD) level 262 (passage 45) were seeded at a density of 4000 cells $/ \mathrm{cm}^{2}$ in culture flasks in Dulbecco's Modified Essential Medium (DMEM; Invitrogen, Life Technologies, Carlsbad, CA) containing 10\% fetal bovine serum (FBS; Invitrogen) and cultivated in a humidified atmosphere of $37^{\circ} \mathrm{C}$ and $5 \% \mathrm{CO}_{2}$. After one week, cells were washed in PBS, detached with $0.125 \%$ trypsin and $5 \mathrm{mM}$ EDTA (Sigma-Aldrich) in PBS, reseeded, and cultured for another week. Cells were trypsinized (PD level 271, passage 47) and resuspended for use $\left(2 \times 10^{7}\right.$ cells $\left./ \mathrm{mL}\right)$ in DMEM/10\% FBS penicillin (100 U/mL; Sigma-Aldrich) and streptomycin (100 mg/L; Sigma-Aldrich). 
The hMSC-TERT cells were seeded onto the top of the scaffolds by pipetting $50 \mu \mathrm{L}$ of cell suspension media with $1 \times 10^{6}$ cells onto each scaffold. The scaffolds were placed in agarose-coated $\left(1 \%\right.$ in sterile $\left.\mathrm{H}_{2} \mathrm{O}\right)$ six-well plates (4 scaffolds/well), and incubated for 2 hours in an incubator. Thereafter, additional $7.5 \mathrm{~mL}$ of DMEM/10\% FBS, $100 \mathrm{U} / \mathrm{mL}$ penicillin, $100 \mathrm{mg} / \mathrm{L}$ streptomycin were added to each well. After 24 hours, cell/scaffold constructs were moved to $58 \mathrm{~mm}$ diameter dual side-arm spinner flasks (Bellco Glass, Vineland, NJ). An autoclavable stainless framework with four needles was constructed and placed in the spinner flasks. Two cell-seeded scaffolds were mounted on each needle giving a total of eight scaffolds per flask. Spinner flasks containing $120 \mathrm{~mL}$ of media were placed on a Bell-ennium ${ }^{\mathrm{TM}}$ five-position magnetic stirrer (Bellco Biotechnology, Vineland, NJ) at 30 revolutions per minute in the incubator with side arm caps loosely attached.

Cell/scaffold constructs were cultured with DMEM/10\% FBS for the first week, and then the medium was replaced with osteogenic stimulation medium (DMEM/10\% FBS with $100 \mathrm{nM}$ dexamethasone, $290 \mu \mathrm{M}$ ascorbic acid and $5 \mathrm{mM}$ $\beta$-glycerophosphate) (all from Sigma-Aldrich) and cultured for up to 21 days. Medium was exchanged twice a week.

\section{Cellular adhesion, viability and proliferation of hMSC-TERT cellular scaffolds \\ Scanning electron microscope (SEM)}

Scaffolds from day 1 , day 7 , day 14 , and day 21 were rinsed in PBS and fixed in $2.5 \%$ glutaraldehyde containing $0.1 \mathrm{M}$ sodium cacodylate buffer ( $\mathrm{pH}$ 7.4) and dehydrated in a graded ethanol series, air-dried. The samples from day 21 with cell culture and day 0 without cell culture were viewed using environmental mode SEM (Nova NanoSEM 600; FEI Company, Hillsboro, OR) and the element component of the crystal-like structure was analyzed by means of an energy dispersive X-ray spectrometer (EDX).

\section{Confocal imaging}

To assess cell viability, the cell/scaffold constructs were incubated for 30 minutes in DMEM containing $10 \mu \mathrm{M}$ CellTracker $^{\mathrm{TM}}$ Green CMFDA (Invitrogen). The staining medium was then replaced with fresh DMEM/10\% FBS and incubated for another 30 minutes at $37^{\circ} \mathrm{C}$. Non-fluorescent CMFDA was converted to a bright green fluorescent product when cytosolic esterases cleaved off the acetates. The cell/ scaffold constructs were then rinsed in prewarmed PBS, fixed in $10 \%$ formalin for 5 minutes at room temperature, and stained with $1 \mu \mathrm{g} / \mathrm{mL}$ Hoechst 33258 (Sigma-Aldrich) in PBS for 20 minutes. Living cells were labeled with green pixels. Nuclei of the cells were stained with Hoechst, labeled with red pixels. Chitosan were stained with yellow pixels resulting from the spatial overlap of red and green pixels. Images were acquired using a laser scanning confocal microscope, 510 Meta (Zeiss Microimaging GmbH, Jena, Germany). The confocal settings (excitation, laser power, detector gain, and pinhole size) were the same for all cell imaging. Separate channels and filters were used. Excitation/emission wavelengths were $488 \mathrm{~nm} / \mathrm{BP} 505-530 \mathrm{~nm}$ for CellTracker ${ }^{\mathrm{TM}}$ Green and $405 \mathrm{~nm} / \mathrm{LP} 420 \mathrm{~nm}$ for Hoechst.

\section{DNA quantification}

The total cell number in the 3D cellular scaffold was estimated by quantifying the dsDNA content in each scaffold using the Quant-iT ${ }^{\text {TM }}$ PicoGreen ${ }^{\circledR}$ dsDNA assay (Invitrogen). Scaffolds were thawed and sonicated at intervals of 1 second on $/ 5$ seconds off for a total of 1 minute. Three milligrams of collagenase (Sigma-Aldrich,) were added to each DNA sample and the samples were incubated in a $37^{\circ} \mathrm{C}$ water bath for 3 hours. One mg proteinase K (SigmaAldrich) was then added and the samples were incubated overnight in a $45^{\circ} \mathrm{C}$ water bath. Sample volume was diluted 1:10 in a Tris-EDTA buffer and vortexed in order to release DNA from scaffold debris. From each sample, $2 \times 50 \mu \mathrm{L}$ were drawn, $50 \mu \mathrm{L}$ of PicoGreen (diluted 1:200 in TE buffer) was added, then the mixture was incubated in darkness for 5 minutes and measured into a 96-well plate using a microplate reader, Victor3 1420 Multilabel Counter, (PerkinElmer, Waltham, MA). Samples were excited at $480 \mathrm{~nm}$, and the fluorescence emission intensity was measured at $520 \mathrm{~nm}$. Standards were prepared according to the manufacturer's instructions (lambda DNA, concentration range: $0-1 \mu \mathrm{g} / \mathrm{mL}$ ). Technical duplicates were used for each biological sample $(n=4)$.

\section{Osteogenic differentiation and mineralization of hMSC-TERT cells in a 3D scaffold Alkaline phosphatase (ALP) activity assay} ALP activity was determined using a colorimetric endpoint assay measuring the enzymatic conversion of p-nitrophenyl phosphate (Sigma-Aldrich) to the yellowish product, p-nitrophenol, in the presence of ALP. p-Nitrophenol absorbance was measured by means of a microspectrophotometer (Victor 1420; Perkin Elmer) at double wavelengths of $405 \mathrm{~nm}$ and $600 \mathrm{~nm}$. Standards were prepared from p-nitrophenol 
(concentration range: $0-0.2 \mathrm{mM}$ ). Technical duplicates were used for each biological sample $(n=4)$.

\section{von Kossa staining}

The scaffolds were rinsed with PBS and fixed for 5 minutes in $4 \%(\mathrm{w} / \mathrm{v})$ formaldehyde solution $(\mathrm{pH} 7.0)$, then washed with $\mathrm{ddH}_{2} \mathrm{O}$, incubated in darkness with a $2.5 \%$ silver nitrate solution for 20 minutes, and subsequently developed by adding $0.5 \%$ hydroquinone for 2 minutes. Finally, surplus silver was removed using sodium thiosulphate for 5 minutes. The scaffolds were dried under vacuum and pictures were taken afterwards.

\section{Calcium content assay}

Calcium contents of cell-seeded scaffolds were quantified using a colorimetric endpoint assay based on the complexation of one $\mathrm{Ca}^{2+}$ ion with two Arsenazo III molecules to a blue-purple product (Diagnostic Chemicals Limited, Charlottetown, PEI, Canada). The calcium deposition was dissolved in $1 \mathrm{M}$ acetic acid by placing it in a shaker overnight. The samples were diluted 1:50 with $\mathrm{ddH}_{2} \mathrm{O}$ and aliquots of $20 \mu \mathrm{L}$ were transferred to a 96-well plate. Arsenazo III solution $(280 \mu \mathrm{L})$ was added and incubated for 10 minutes at room temperature. A standard dilution series of calcium ranging from 0 to $50 \mu \mathrm{g} / \mathrm{mL}$ was prepared and $\mathrm{Ca}^{2+}$ concentration was quantified spectrophotometrically at $650 \mathrm{~nm}$. Calcium content was expressed as micrograms of $\mathrm{Ca}^{2+}$ per scaffold.

\section{Histology and immunohistochemistry}

The scaffolds were fixed in $70 \%$ ethanol, Technovit ${ }^{\circledR} 7100$ (Ax-lab, Vedbæk, Denmark) embedded, and cut into $25 \mu \mathrm{m}$ sections using a Sawing Microtome KDG 95 (Meprotech, Dirkshorn, the Netherlands). Sections were taken from the peripheral and the central part of the scaffold. Hematoxylin and eosin staining was applied in order to reveal cell distribution. Histochemical staining for ALP was performed to test the osteogenic phenotype of cells cultured in the scaffolds. For immunohistochemistry, the sections were incubated overnight with rabbit anti-human osteocalcin antibody (BT593; Biomedical technologies Inc, Stoughton, MA), followed by biotinylated goat antirabbit IgG (E0432; DAKO, Glostrup, Denmark) for 1 hour, and peroxidase-conjugated streptavidin (P0397; DAKO) for 1 hour. Sections were visualized with 3-amino-9ethylcarbazol (A6926; Sigma-Aldrich) and counter-stained with Mayer's hematoxilin. With the same staining procedure, sections stained without the primary antibody of the rabbit anti-human osteocalcin served as control. Images were photographed using a BX50 microscope with a Camedia C-5060 camera (Olympus, Tokyo, Japan).

\section{Statistical analysis}

Results are presented as mean \pm standard deviation (SD) for $\mathrm{n}=4$ biological replicates. The data of DNA quantification, ALP activity, and calcium content were analyzed by one-way analysis of variance (ANOVA) using the Statgraphics Centurion XVI software version 16.1.05 (Statpoint Technologies, Inc, Warrenton, VA). Data were tested for normal distribution and variance homogeneity using Levene's-test. Multiple range test (LSD 95\%) was used to identify differences between sampling days at the $5 \%$ significance level.

\section{Results \\ Drug release from scaffolds}

DOX without modified clay, DOX with modified clay, and clay/DOX carrier were each incorporated into individual scaffolds. The release profile of DOX from these three different composite scaffolds is shown in Figure 2. There was an initial burst release in all the groups. On day 4, DOX released $94 \%$ of the total amount of drug from the Group B control scaffolds (prepared without modified clay). Whereas, the clay incorporated scaffolds released far less amounts of drug loaded: 13\% from Group C scaffolds (prepared by direct mixing with modified clay and DOX) and 15\% from Group D scaffolds (prepared with clay/DOX carrier). The cumulative drug release was significantly lower from Group C scaffolds than Group D scaffolds on day $5(P=0.04)$. By day 56 , about $33 \%$ was released by Group C scaffolds and 47\% was released by Group D scaffolds.

\section{Cell adhesion, viability and proliferation in the scaffold}

Scanning electron microscopy (Figure 3A) shows the cells and extracellular matrix deposition on the scaffolds. On day 1 , the cells anchored tightly on the surface of the scaffold. Cells were adhered and spread well on the scaffold. On day 7, cells and extracellular matrix deposition partially covered the scaffold. Increasing density and extracellular matrix deposition almost completely covered the scaffold on day 14. Crystal-like extracellular matrix deposition was observed on the surface of the scaffold of the 21 day culture. These deposits were expected to be calcium phosphate and were further identified by element component analysis (energy dispersive X-ray spectrometer) to consist mainly of 


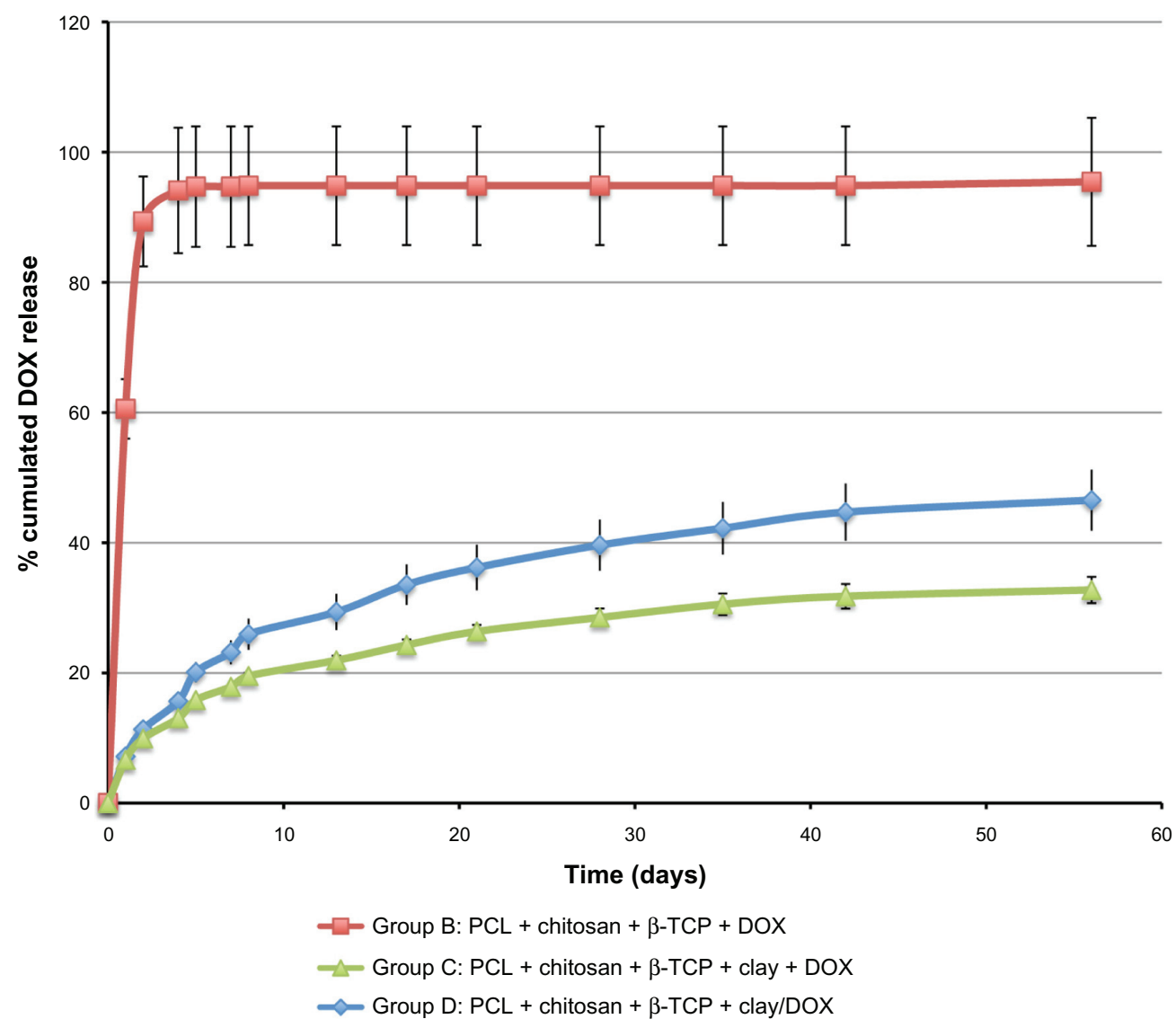

Figure 2 Cumulative DOX release (\%) from Group B (DOX without clay scaffold), Group C (DOX with clay scaffold), and Group D (Clay/DOX carrier scaffold) at $37^{\circ} \mathrm{C}$ in PBS (pH 7.4) for 2 months.

Abbreviations: PCL, polycaprolactone; $\beta$-TCP, $\beta$-tricalcium phosphate; DOX, doxorubicin hydrochloride; PBS, phosphate-buffered saline.

$\mathrm{P}, \mathrm{Ca}$, and O. Compared to the scaffold without cell culture on day 0 , the amount of calcium increased significantly.

Confocal microscopy images (Figure 3B) showed good cell viability in the scaffolds during the 21 days of culturing. Cells attached and spread in the scaffolds from day 1. Cells migrated into the macro- and micropores of the scaffolds and spread evenly on the surface of the scaffolds. Cell density increased steadily as the culturing period progressed. Higher magnification showed that the cells grew into the chitosan structure and proliferated rapidly (the nuclear density increased).

The DNA amount was assumed to be proportional to the cell number. Thus, cell proliferation over time could be followed by quantification of the extracted DNA from the scaffolds. DNA amounts increased during the culturing period (Figure 3C).

\section{Osteogenic differentiation} and mineralization of hMSC-TERT cells in the 3D scaffold

ALP activity was highest on day 7, then decreased at day 14 , after which the same level was maintained until day 21 .
This suggests that the cells started to initiate mineralization on day 7 (Figure 4A).

ALP positive staining (brown color) confirmed the presence of ALP, which was a component and marker for extracellular matrix produced by osteogenic differentiated cells (Figure 4B).

Quantitative data of calcium content (Figure 4C) and von Kossa staining (Figure 4D) showed that the scaffolds were osteoinductive.

\section{Histology}

Cross-sections of the scaffold with hematoxylin and eosin staining revealed the cellular distribution within the scaffold (Figure 5A). Nuclei were stained dark blue (basophilic), extracellular matrix and cytoplasm were stained purple, and the chitosan foam was stained orange. Cells migrated into the center of the scaffolds within 7 days of culture and the pores of the scaffolds were partly filled with cells and extracellular matrix. The depth of cell infiltration and the density of the cells increased as culturing progressed. On day 21, cells had fully filled the macro- and micropores of the scaffolds. 

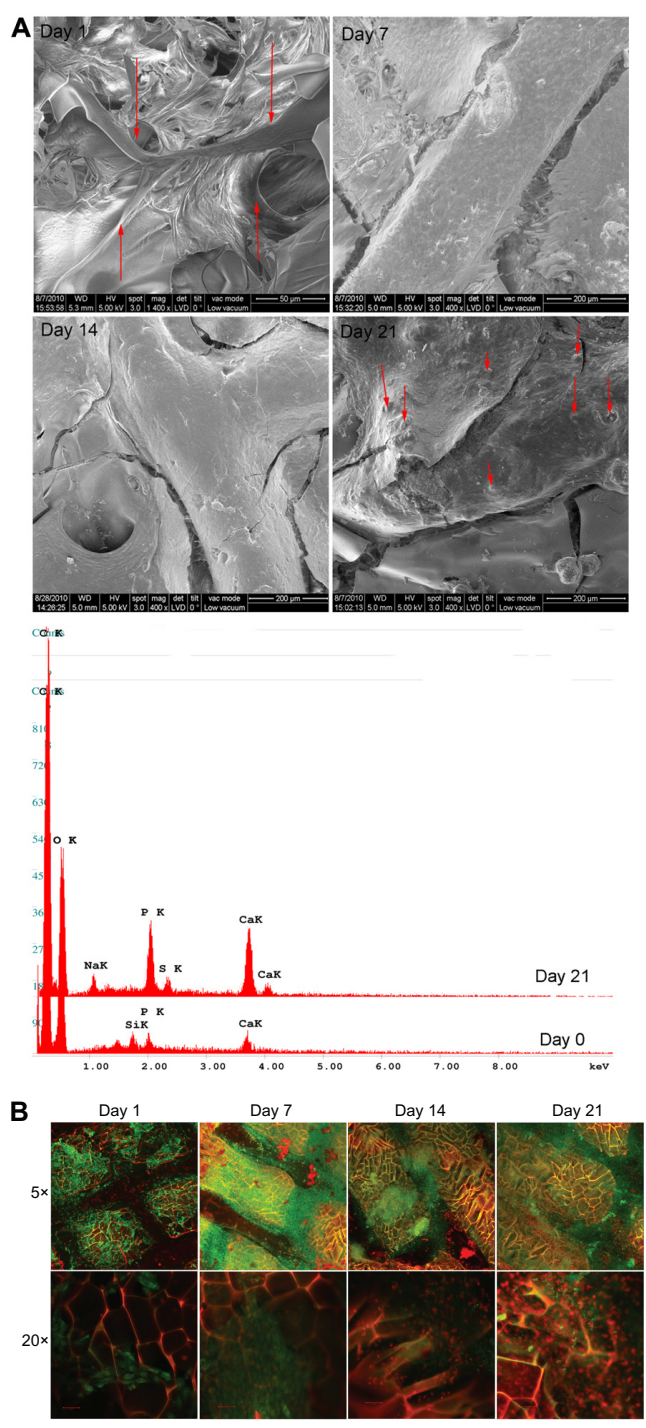

C

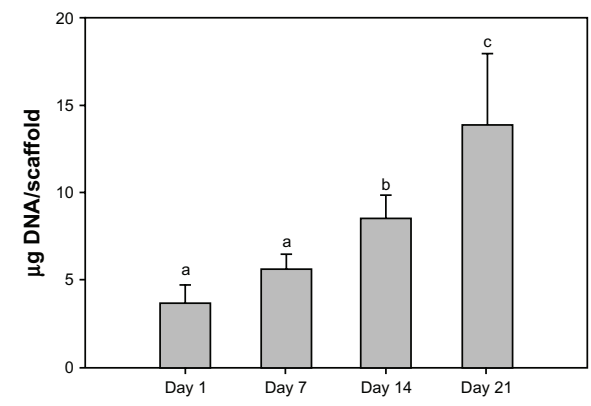

Figure 3 Cell adhesion, viability, and proliferation in the scaffolds. (A) SEM images of cellular scaffolds from the cell seeding side on Days I, 7, 14, and 2I. The arrows point to the cells on Day I and to the crystal-like extracellular matrix deposition on Day 21. EDX analysis of the element components revealed the mineralized nodules as calcium phosphate salts, consisting of $\mathrm{P}, \mathrm{Ca}$, and $\mathrm{O}$ elements in the cell culture scaffold for Day 21 , compared to day 0 in the cell free scaffold. (B) Confocal micrographs of cell viability on Days I, 7, 14, and 2I. Green pixels: live cells; red pixels: nuclei; green and red pixel overlap: chitosan structure. Upper panels show projections of a $150 \mu \mathrm{m}$ optical section onto a single plane (magnification $5 \times$ ). Bottom panels show single planes at magnification 20x. (C) Quantification of dsDNA in cellular scaffolds by means of PicoGreen assay on Days I, 7, I4, and 21 .

Notes: The amount of DNA is expressed as mean \pm SD $(n=4)$. Different letters $(a-c)$ indicate significant differences $(P<0.05)$ between sampling days.

Abbreviations: ALP, alkaline phosphatase; SEM, scanning electron microscopy; EDX, energy dispersive X-ray spectrometer.
Positive osteocalcin staining (red dots, pointed with arrows) showed that hMSCs-TERT cells had undergone osteogenic differentiation and secreted bone-related extracellular matrix marker-osteocalcin (Figure 5B).

\section{Discussion}

We fabricated a drug-eluting scaffold consisting of two main parts: a rapid prototyped PCL scaffold for mechanical support and an embedded chitosan/nanoclay/ $\beta$-TCP composite for sustained drug delivery and enhancement of osteogenesis. This composite scaffold had a favorable environment for cell attachment, proliferation, and osteogenic differentiation of hMSCs. This scaffold also had the capacity to load and release an anticancer drug in a sustained manner. The present study suggests that the composite scaffold has the potential to be a drug-eluting bone graft substitute.

In the clinic, tumor resection is followed by systemically administered chemotherapy to prevent tumor recurrence. The limitations of systemic drug administration are limited bioavailability at the tumor site, systemic toxicity, and other adverse effects. Although systemically administered tumor-targeted drug delivery has been a great focus of advanced drug-delivery research, no breakthrough products for clinical use are as yet on the market. At present, local sustained drug release from an implanted device at the tumor site is a more realistic approach. The Gliadel ${ }^{\circledR}$ Wafer (Eisai Inc., Tokyo, Japan) is an early example of a sustained drug delivery device that can be implanted locally as an adjunct to surgery for malignant glioma. Various local drug-delivery systems for different tumor types have been researched. Itokazu et al showed that methotrexate released from porous hydroxyapatite blocks and $\beta$-TCP blocks remained effective against tumor cells for up to 12 days in vitro. ${ }^{33}$ El-Ghannam et al tested a ceramic-based anticancer drug to treat breast cancer in a murine model. ${ }^{34}$ However, it was difficult to tune the drug amount and release kinetic in those systems.

Many studies have used clay to enhance the solubility of poorly soluble drugs and to promote sustained release. Ofloxacin has been controllably released from chitosan/clay hydrogel beads. ${ }^{21}$ A pseudo-zero-order release kinetics of vitamin B12 was observed from clay/chitosan nanohydrogel. ${ }^{22}$ Wang et al showed that BSA could be controllably released from clay nanoparticles. ${ }^{35}$ However, the ultimate goals of all these studies were to use clay or clay composite as a simple drug carrier rather than as a component in an implantable device. Therefore, we included the montmorillonite clay in the drug-eluting matrices in this study. 

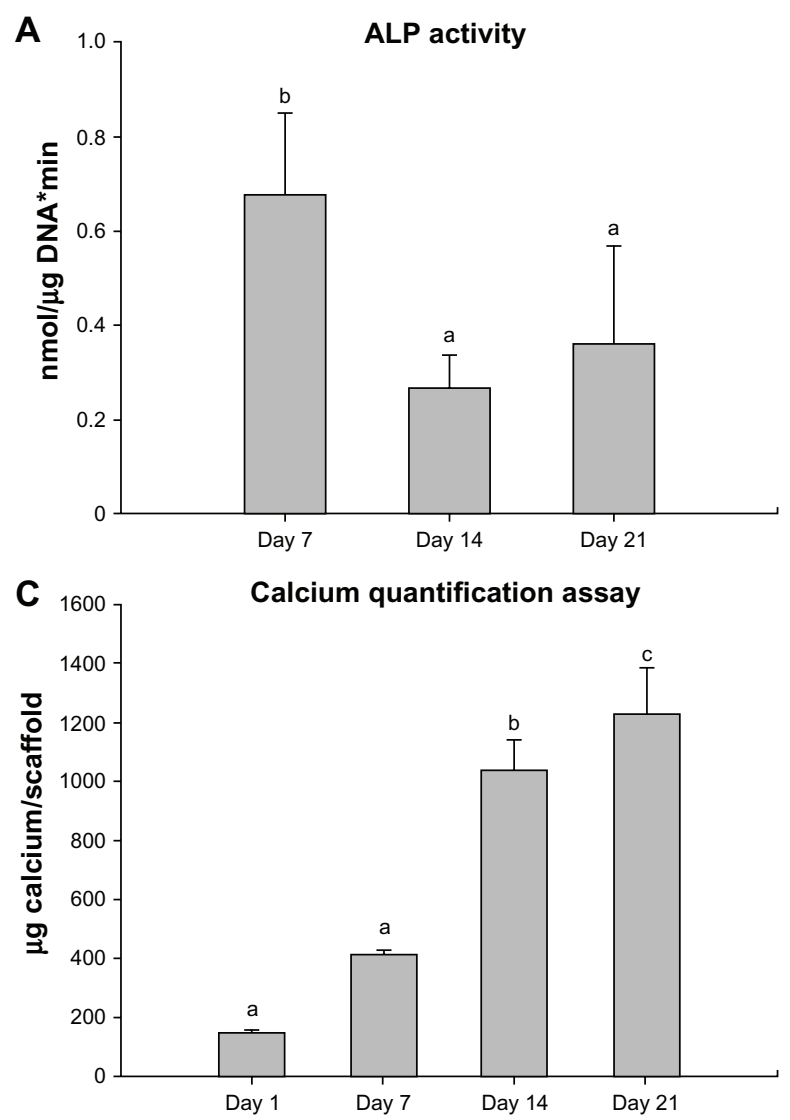

B

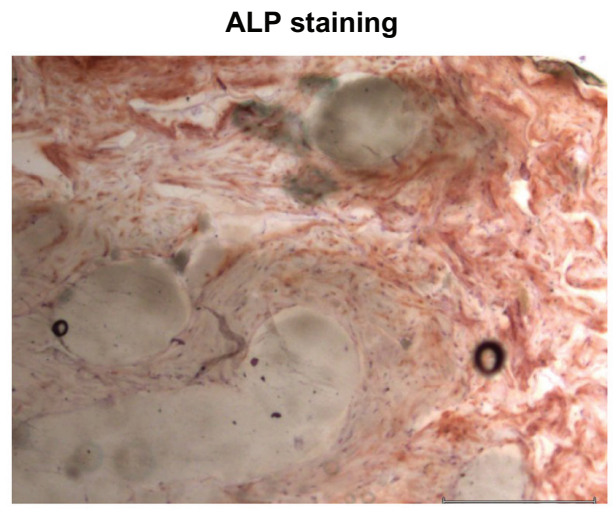

D

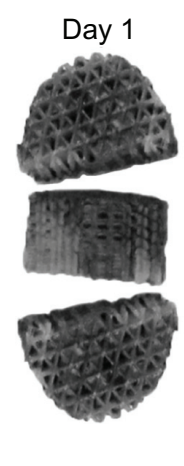

von Kossa staining

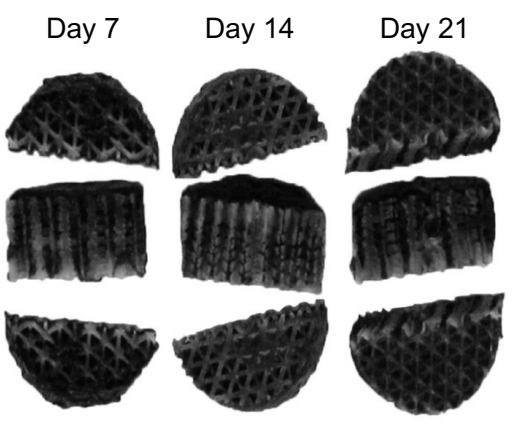

Figure 4 Osteogenic differentiation and mineralization of cellular scaffolds. (A) Activity of the ALP enzyme on Days I, 7, I4, and 2 I. The activity is expressed as mean \pm SD $(n=4)$. Activity is indicated in nanomole $p$-nitrophenol/microgram DNA per minute ( $\mathrm{nmol} / \mu \mathrm{g}$ DNA*min). Different letters $(\mathrm{a}-\mathrm{c})$ indicate significant differences $(P<0.05)$ between sampling days. (B) ALP staining. (C) Calcium contents per scaffold on Days I, 7, I4, and 2 I. The amount of calcium is expressed as mean \pm SD ( $\mathrm{n}=4$ ). Different letters $(\mathrm{a}-\mathrm{c})$ indicate significant differences $(P<0.05)$ between sampling days. (D) von Kossa staining of scaffolds.

Abbreviations: ALP, alkaline phosphatase; SD, standard deviation.

We chose sodium montmorillonite due to its high cation exchange capacity, good absorbance, and drug carrying capability. The structure of montmorillonite clay consists of one shared edge of an octahedral sheet of aluminum hydroxide fused in between two silica tetrahedrals. ${ }^{36}$ It has a plate-like layer with the thickness of $1 \mathrm{~nm}$ and a high aspect ratio (ratio of length to thickness), which gives it a large surface area and makes it suitable for reinforcement purposes. These layers can stack and lead to a regular van der Waal's gap between layers. In the interlayer region, there exist exchangeable cations such as $\mathrm{Na}^{+}$and $\mathrm{Ca}^{2+}$, which enable intercalation with drugs and polymers.

The principle of our composite scaffold with drug-eluting matrix embedding is shown in Figure 6. The experiment rationale of the designed composite scaffold is two-fold: firstly, to show the successful loading and release of a chemotherapeutic drug, doxorubicin, and secondly, to test the drug-free composite for bone tissue repair using hMSC. All the tests were done in vitro as shown in Figure 1. The drug was released sustainably from the composite scaffolds for 2 months. About $45 \%$ DOX was released after 56 days from the scaffold when clay was incorporated into a chitosan/ $\beta$-TCP matrix. In contrast, about $95 \%$ DOX was released within 4 days from the scaffolds without clay. This confirms that clay is an effective material in drug delivery modulation.

Even with the same amount of modified clay in the scaffolds, a faster drug-release rate was observed when clay/DOX carriers were first prepared and mixed in the chitosan/ $\beta$-TCP solution (Figure 2, Group D). A slower drug-release rate was observed when the drug was directly mixed with modified clay in the chitosan/ $\beta$-TCP solution during the scaffold preparation (Figure 2, Group C). It is postulated that DOX intercalates into clay layers by replacing $\mathrm{Na}^{+}$. The pKa of DOX is around 8.3 and the drug loaded composites are prepared in acidic $\mathrm{pH}$. Under the acidic $\mathrm{pH}$, the drug molecules will be positively charged. When DOX and clay were directly mixed with the chitosan/ $\beta$-TCP solution, DOX $(\mathrm{pKa} \sim 8.3)$ and chitosan $(\mathrm{pKa} \sim 6.3)$ competitively replaced $\mathrm{Na}^{+}$ions 
A
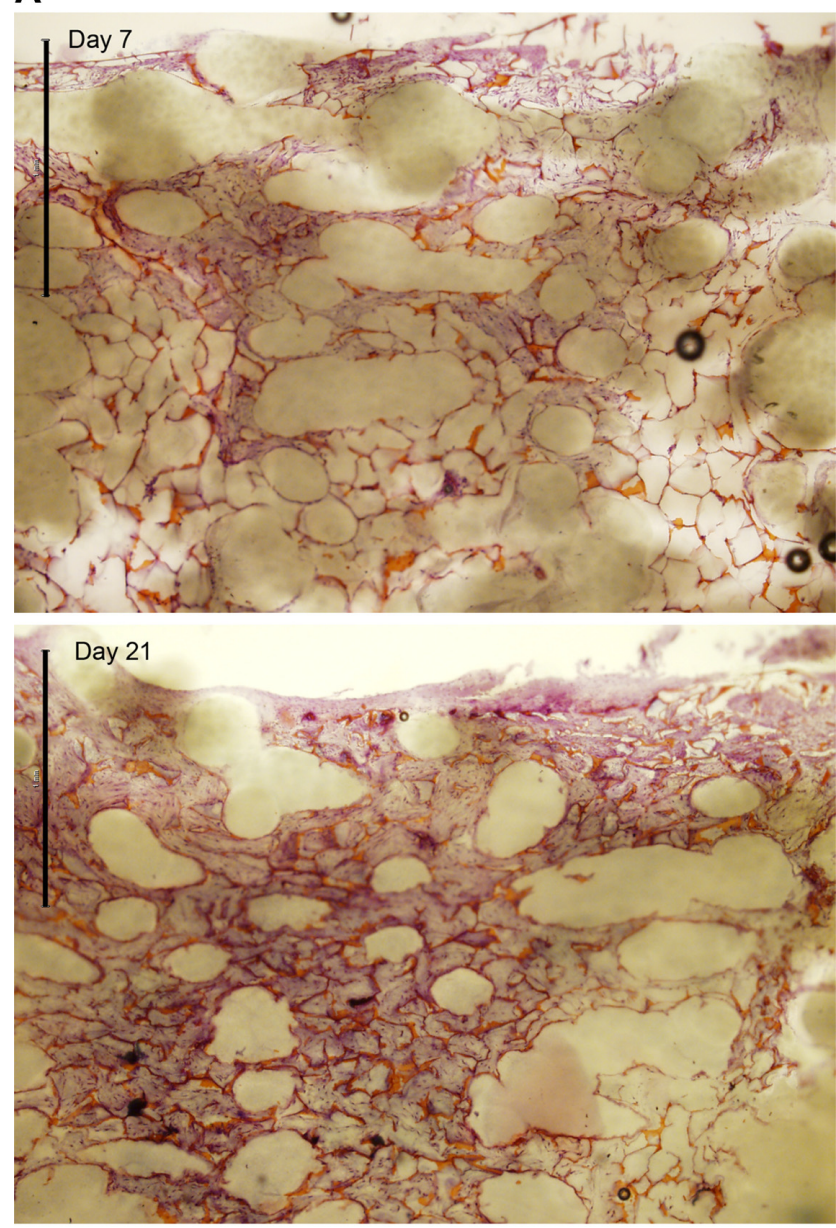

B
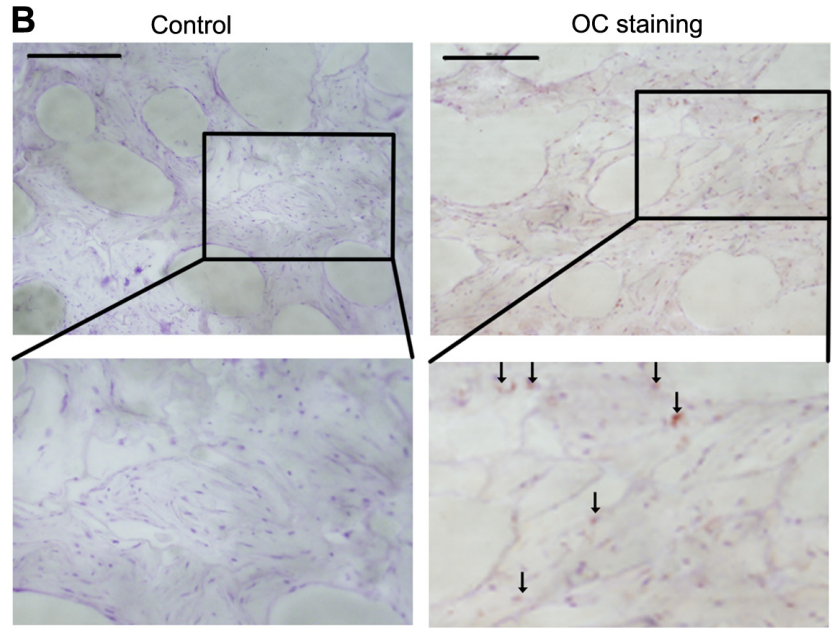

Figure 5 Histology results. (A) Representative histology micrographs of H\&E stain showing cross-sections of the top part of cell/scaffold constructs after 7-day and 21-day culture. Bars $=1 \mathrm{~mm}$. Nuclei are stained dark blue (basophilic), ECM and cytoplasm are stained purple, and the chitosan foam is stained orange. (B) Immunohistology staining of anti-human osteocalcin of the scaffolds after 2I-day culture. Sections stained without anti-human osteocalcin served as control.

Note: Arrows point to representative positive staining of osteocalcin.

Abbreviations: OC, osteocalcin; H\&E, hematoxylin and eosin; ECM, extracellular matrix.
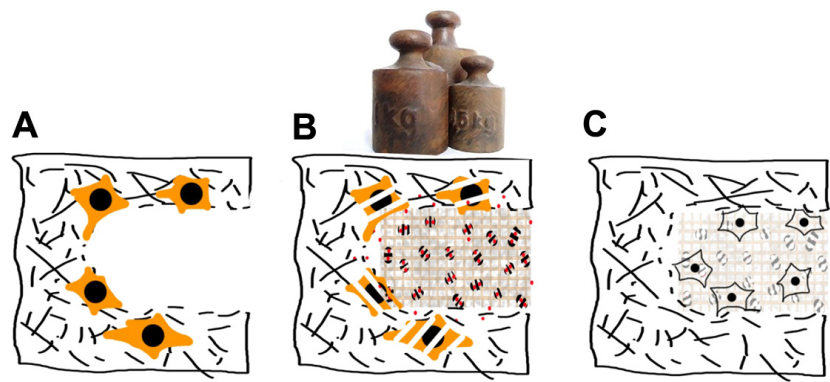

Figure 6 Principle of the composite scaffold with drug eluting matrix embedding. (A) Following tumor resection, remnant tumor cells (orange) may be present and there is an increased risk of recurrence. (B) Implantation of mechanically stable porous scaffold with controlled drug release functions will provide immediate load bearing support and localized anti-cancer treatment. (C) After all the drug has been released, the implant will aid in tissue regeneration of the wound site.

in the clay layers. The smaller and more positively charged DOX could replace more $\mathrm{Na}^{+}$ions than chitosan. As a result, the binding affinity with clay and DOX was stronger and release was slower in Group C scaffolds. Yuan et al also showed that DOX release from clay was much slower than from a chitosan/clay composite carrier. ${ }^{23}$ When the drug was loaded to a chitosan/clay nanocomposite prior to the composite preparation as in Group D scaffolds, no competitive intercalation and exfoliation of the clay was expected. Therefore, the drug-release rate was faster as a low affinity of DOX to clay was anticipated.

Therefore, tunable drug-release rates from the scaffold can be created by adjusting the amounts and types of chitosan in chitosan-clay composite preparations. The ratios between clay and chitosan of the composites could accomplish the same result. A study by Hua et al showed that increasing the ratio of clay to chitosan enhanced drug entrapment and reduced drug release. ${ }^{21}$ Similar effects were observed in biopolymer/clay nanocomposites. ${ }^{35}$ These studies indicated that drug-release kinetics could be adjusted by altering clay/chitosan/drug ratios and compositions in our composite scaffolds.

For biomedical applications, Katti et al reported that a novel chitosan/clay/hydroxyapatite sheet is biocompatible and, in comparison to pure chitosan as well as chitosan/ hydroxyapaptite and chitosan/clay, possesses improved mechanical properties. ${ }^{24}$ In another study, they showed that chitosan/polygalacturonic acid scaffolds containing modified montmorillonite clay appeared to satisfy some of the basic requirements of scaffolds for bone tissue engineering applications. ${ }^{25}$ Chitosan/clay nanocomposites are also potential sustained drug-release carriers. ${ }^{21-23}$

The second objective of the study was to test if the drugfree composite scaffold (or drug-depleted scaffold in a clini- 
cal setting) is suitable for bone repair, because patients need bone grafts or artificial bone implants to be replaced at the resected tissue in order to provide immediate mechanical support and bone regeneration. In this study, we chose the rapid prototyped PCL scaffold to house the chitosan/clay/ $\beta$-TCP composite because the rapid prototyped scaffolds can be fabricated to resemble the shape and mechanical strength of bone. ${ }^{37}$ The intertwined network of the chitosan/nanoclay/ $\beta$ TCP composite was designed to provide better biocompatibility and osteogenesis. Calcium phosphates like $\beta$-TCP and hydroxyapatite were widely applied as coatings on other implants like titanium to achieve faster and greater bone ingrowth. ${ }^{38,39}$ Chitosan has also been widely investigated for bone tissue engineering and drug delivery because of its favorable biological properties including biocompatibility, biodegradability, nontoxicity, osteoconductivity, and antibacterial properties. ${ }^{40}$ However, both $\beta$-TCP and chitosan have lacked the necessary mechanical properties to mimic bone because $\beta$-TCP is brittle and porous chitosan scaffolds showed inferior tensile and compressive strength in comparison to natural bone ${ }^{41-43}$ Clay is a silicate compound, a class of ceramics that is gaining increasing interest in biomedical applications. ${ }^{44}{ }^{46}$ Katti et al showed that a nanocomposite sheet of chitosan/clay/hydroxyapatite was biocompatible and had significantly improved nanomechanical properties. ${ }^{24}$

We cultured hMSCs-TERT cells in our scaffolds and observed high cell viability and cell infiltration, confirmed by SEM, confocal microscopy, and histology. In particular, a very highly increased $\mathrm{Ca}^{2+}$ deposition rate was observed compared to our first study with hyaluronic acid and methylated collagen. ${ }^{47}$ The $\mathrm{Na} \rightarrow \mathrm{Ca}$ exchange equilibrium constant for sodium montmorillonite is close to $1,{ }^{48}$ so when found in cell culture media or blood plasma, which contains approximately 60 times more sodium than calcium, the majority of metal cations in the clay would be $\mathrm{Na}^{+}$. Chitin, chitosan, and their derivatives readily bind to divalent cations, with particular affinity for heavy metal ions but still including $\mathrm{Ca}^{2+}{ }^{29-51}$ This chelation property has been studied extensively for use in wastewater treatment. Rats fed with chitosan-enriched diets have decreased mineral absorption with a resulting decrease in bone quality. ${ }^{52}$ Consequently, we performed a control experiment with cell-free scaffolds in similar cell culture media and measured $\mathrm{Ca}^{2+}$ deposition for 21 days (Figure 7).

Our suspicions were confirmed, as the cell-free scaffolds had a similar amount of calcium deposition comparable to the cell-seeded scaffolds up to day 7 and had nearly two times the amount of calcium at day 14 and three times at

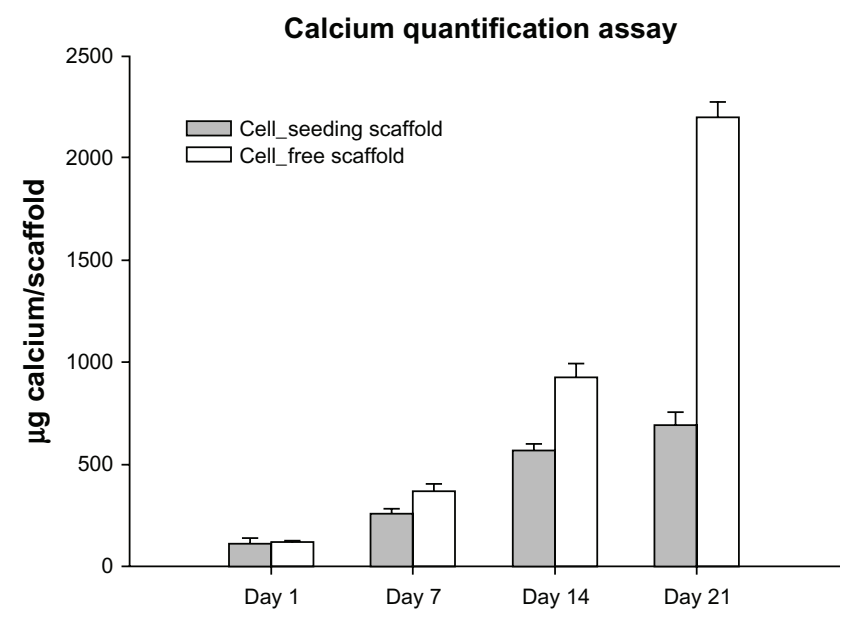

Figure 7 Calcium deposition contents per scaffold on cell-free scaffolds in comparison with cell-seeded scaffolds on Days I, 7, 14, and 21 .

day 21 compared to the cell-seeded scaffolds. The increasing progression of the graph can be explained by the regular media change with corresponding replenishment and further binding of $\mathrm{Ca}^{2+}$ in the scaffold. Dynamic culture and the large surface area of the chitosan foam have most likely been major contributors to the thorough accumulation of calcium. As seen in Figure 5A, the slowed calcium deposition in the cell-seeded scaffolds coincides with the increasing cellularity, which decreases the exposed surface area of the chitosan foam inside the scaffold and decreases metabolite and ion exchange rate by obliterating the scaffold pores.

Numerous papers in bone tissue engineering have studied the biocompatibility of chitosan scaffolds in vitro and used calcium assays and von Kossa staining to conclude the osteoinductive capability of the material..$^{53-56}$ The majority of these studies do not show mineralization data from cell-free controls. As seen in this study, although chitosan is clearly highly biocompatible and osteoconductive, ${ }^{40,57,58}$ the osteoinductive potential of this particular ionotropic biomaterial should not be evaluated only by the calcium deposition. We included an immunostaining against osteocalcin (Figure 5B) to qualitatively demonstrate osteogenic differentiation in the scaffold.

With the same amount of seeding cells, the measured DNA content is lower than that of the scaffold in the first study using hyaluronic acid and methylated collagen. ${ }^{47}$ This could be due to inefficient extraction of DNA in the presence of a cationic polymer like chitosan. Chitosan readily forms complex coacervates with free DNA, which makes it useful for making DNA-chitosan nanoparticles for drug delivery. ${ }^{59}$ It is unlikely that the clay contributed to DNA retention, as its absorption of polycations at physiological $\mathrm{pH}$ is minimal. ${ }^{60}$ 
Therefore, Picogreen used for DNA quantification cannot intercalate a DNA-chitosan complex and an underestimated value is to be expected.

ALP quantification measures the activity, ie, the amount of a protein macromolecule in the purified supernatant, and should not be affected by the adsorption and chelation properties of clay and chitosan.

Therefore, the optimal combination of four biomaterials (PCL, chitosan, $\beta$-TCP, and clay) will potentially prove to be a much needed contribution in terms of filling a vital gap in the field of therapeutic implant. Further in vivo studies on this composite scaffold are underway as the more realistic conditions for bone repair occurred after the release of chemotherapeutic drugs.

Although it is mere speculation at this juncture, further development of the therapeutic implant can be envisioned from this work. The concept of using rapid prototyped PCL as a biocompatible structural support, and soft clay composites as a drug reservoir, can be extended for the treatment of different tissues that require local sustained drug release. The only limitation will be the choice of polymer for effective dispersion of clay. The composite has to be reproducible for both sustained drug delivery and tissue repair. Other naturally derived polymers, such as alginate and gelatin, will also be good candidates for preparation of the composite. Instead of a cation exchanger like sodium montmorillonite, an anion exchanger can also be applied in this system for carrying different properties of drugs. In this case, a different class of clays, layered double hydroxides, would be used. Since the amount and type of drug needed for different patients vary from subject to subject and the severity of the medical implications, personalized therapeutic implants are necessary. Designing a composite scaffold based on the concept of this work will further contribute to the development of personalized medical care.

\section{Conclusion}

We fabricated a 3D hybrid scaffold composed of two main parts: a rapid prototyped PCL scaffold for mechanical support and chitosan/clay/ $\beta$-TCP for enhanced bone repair and local sustained drug delivery. The composite scaffold design offered a favorable environment for cell attachment, proliferation, and osteogenic differentiation of hMSC-TERT. The developed scaffold could provide a sustained drug release of the loaded doxorubicin. Doxorubicin was used in this study as a model drug to demonstrate the release kinetic of the drug from the scaffold. The tunable characteristic of clay composite to carry drug was also explained based on the extent of intercalation in clay. By applying the concept of this scaffold design, local sustained drug-release tissue engineering scaffolds can be developed for the treatment of diseases in other tissues.

\section{Acknowledgments}

This study was financially supported by the Lundbeck Foundation, the Danish Research Council, and Aarhus University Research Foundation. We would like to thank Anette Baatrup, Anna Bay Nielsen, and Lisa Feng for their technical assistance. This paper was accepted as a poster presentation at the 57th Annual Meeting of the Orthopedic Research Society, Long Beach, USA, 2011.

\section{Disclosure}

The authors report no conflicts of interest in this work.

\section{References}

1. Sohier J, Daculsi G, Sourice S, de Groot K, Layrolle P. Porous beta tricalcium phosphate scaffolds used as a BMP-2 delivery system for bone tissue engineering. J Biomed Mater Res A. 2010;92(3): 1105-1114.

2. Huang W, Shi X, Ren L, Du C, Wang Y. PHBV microspheres - PLGA matrix composite scaffold for bone tissue engineering. Biomaterials. 2010;31(15):4278-4285.

3. Meijer GJ, de Bruijn JD, Koole R, van Blitterswijk CA. Cell-based bone tissue engineering. PLoS Med. 2007;4(2):e9.

4. Bumgardner JD, Chesnutt BM, Yuan Y, et al. The integration of chitosan-coated titanium in bone: an in vivo study in rabbits. Implant Dent. 2007;16(1):66-79.

5. Hattori H, Masuoka K, Sato M, et al. Bone formation using human adipose tissue-derived stromal cells and a biodegradable scaffold. J Biomed Mater Res Part B Appl Biomater. 2006;76(1):230-239.

6. Lee KY, Peters MC, Anderson KW, Mooney DJ. Controlled growth factor release from synthetic extracellular matrices. Nature. 2000;408(6815):998-1000.

7. Niu X, Feng Q, Wang M, Guo X, Zheng Q. Porous nano-HA/collagen/ PLLA scaffold containing chitosan microspheres for controlled delivery of synthetic peptide derived from BMP-2. J Control Release. 2009;134(2):111-117.

8. Stallmann HP, Faber C, Bronckers AL, Nieuw Amerongen AV, Wuisman PI. In vitro gentamicin release from commercially available calcium-phosphate bone substitutes influence of carrier type on duration of the release profile. BMC Musculoskelet Disord. 2006;7:18.

9. Defail AJ, Edington HD, Matthews S, Lee WC, Marra KG. Controlled release of bioactive doxorubicin from microspheres embedded within gelatin scaffolds. J Biomed Mater Res A. 2006;79(4):954-962.

10. Fröschle GW, Mählitz J, Langendorff HU, Achilles E, Pollock J, Jungbluth KH. Release of daunorubicin from polymethylmethacrylate for the improvement of the local growth control of bone metastasis animal experiments. Anticancer Res. 1997;17(2A): 995-1002.

11. Mouriño V, Boccaccini A. Bone tissue engineering therapeutics: controlled drug delivery in three-dimensional scaffolds. $J R$ Soc Interface. 2010;7(43):209-227.

12. Porter JR, Ruckh TT, Popat KC. Bone tissue engineering: a review in bone biomimetics and drug delivery strategies. Biotechnol Prog. 2009;25(6):1539-1560. 
13. Bose $\mathrm{S}$, Tarafder S. Calcium phosphate ceramic systems in growth factor and drug delivery for bone tissue engineering: A review. Acta Biomater. 2012;8(4):1401-1421.

14. Habraken WJ, Wolke JG, Jansen JA. Ceramic composites as matrices and scaffolds for drug delivery in tissue engineering. Adv Drug Deliv Rev. 2007;59(4-5):234-248.

15. Chawla JS, Amiji MM. Biodegradable poly(epsilon -caprolactone) nanoparticles for tumor-targeted delivery of tamoxifen. Int J Pharm. 2002;249(1-2):127-138.

16. Wenk E, Meinel AJ, Wildy S, Merkle HP, Meinel L. Microporous silk fibroin scaffolds embedding PLGA microparticles for controlled growth factor delivery in tissue engineering. Biomaterials. 2009;30(13): 2571-2581.

17. Hutmacher DW, Sittinger M, Risbud MV. Scaffold-based tissue engineering: rationale for computer-aided design and solid free-form fabrication systems. Trends Biotechnol. 2004;22(7):354-362.

18. McGlashan SA, Halley PJ. Preparation and characterisation of biodegradable starch-based nanocomposite materials. Polym Int. 2003; 52(11):1767-1773.

19. Joshi GV, Kevadiya BD, Patel HA, Bajaj HC, Jasra RV. Montmorillonite as a drug delivery system: intercalation and in vitro release of timolol maleate. Int J Pharm. 2009;374(1-2):53-57.

20. Aguzzi C, Cerezo P, Viseras C, Caramella C. Use of clays as drug delivery systems: Possibilities and limitations. Appl Clay Sci. 2007; 36(1-3):22-36.

21. Hua S, Yang H, Wang W, Wang A. Controlled release of ofloxacin from chitosan-montmorillonite hydrogel. Appl Clay Sci. 2010;50(1): 112-117.

22. Liu KH, Liu TY, Chen SY, Liu DM. Drug release behavior of chitosanmontmorillonite nanocomposite hydrogels following electrostimulation. Acta Biomater. 2008;4(4):1038-1045.

23. Yuan Q, Shah J, Hein S, Misra RD. Controlled and extended drug release behavior of chitosan-based nanoparticle carrier. Acta Biomater. 2010;6(3):1140-1148.

24. Katti KS, Katti DR, Dash R. Synthesis and characterization of a novel chitosan/montmorillonite/hydroxyapatite nanocomposite for bone tissue engineering. Biomed Mater. 2008;3(3):034122.

25. Ambre AH, Katti KS, Katti DR. Nanoclay based composite scaffolds for bone tissue engineering applications. JNanotechnol Eng Med. 2010; 1(3):031013.

26. Poon R, Borys N. Lyso-thermosensitive liposomal doxorubicin: a novel approach to enhance efficacy of thermal ablation of liver cancer. Expert Opin Pharmacother. 2009;10(2):333-343.

27. Müller I, Jenner A, Bruchelt G, Niethammer D, Halliwell B. Effect of concentration on the cytotoxic mechanism of doxorubicin - apoptosis and oxidative DNA damage. Biochem Biophys Res Commun. 1997;230(2):254-257.

28. Young RC, Ozols RF, Myers CE. The anthracycline antineoplastic drugs. N Engl J Med. 1981;305(3):139-153.

29. Antman K, Crowley J, Balcerzak SP, et al. An intergroup phase III randomized study of doxorubicin and dacarbazine with or without ifosfamide and mesna in advanced soft tissue and bone sarcomas. $J$ Clin Oncol. 1993;11(7):1276-1285.

30. Takemura G, Fujiwara H. Doxorubicin-induced cardiomyopathy from the cardiotoxic mechanisms to management. Prog Cardiovasc Dis. 2007;49(5):330-352.

31. Kumar D, Kirshenbaum LA, Li T, Danelisen I, Singal PK. Apoptosis in adriamycin cardiomyopathy and its modulation by probucol. Antioxid Redox Signal. 2001;3(1):135-145.

32. Simonsen JL, Rosada C, Serakinci N, et al. Telomerase expression extends the proliferative life-span and maintains the osteogenic potential of human bone marrow stromal cells. Nat Biotechnol. 2002;20(6):592-596.

33. Itokazu M, Sugiyama T, Ohno T, Wada E, Katagiri Y. Development of porous apatite ceramic for local delivery of chemotherapeutic agents. J Biomed Mater Res. 1998;39(4):536-538.
34. El-Ghannam A, Ricci K, Malkawi A, et al. A ceramic-based anticancer drug delivery system to treat breast cancer. J Mater Sci Mater Med. 2010;21(9):2701-2710.

35. Wang X, Du Y, Luo J. Biopolymer/montmorillonite nanocomposite: preparation, drug-controlled release property and cytotoxicity. Nanotechnology. 2008;19(6):065707.

36. Rhim JW, Ng PK. Natural biopolymer-based nanocomposite films for packaging applications. Crit Rev Food Sci Nutr. 2007;47(4): 411-433.

37. Peltola S, Melchels F, Grijpma D, Kellomäki M. A review of rapid prototyping techniques for tissue engineering purposes. Ann Med. 2008;40(4):268-280.

38. Taché A, Gan L, Deporter D, Pilliar RM. Effect of surface chemistry on the rate of osseointegration of sintered porous-surfaced Ti-6Al-4V implants. Int J Oral Maxillofac Implants. 2004;19(1):19-29.

39. Lickorish D, Ramshaw JA, Werkmeister J, Glattauer V, Howlett CR. Collagen-hydroxyapatite composite prepared by biomimetic process. J Biomed Mater Res A. 2004;68(1):19-27.

40. Di Martino A, Sittinger M, Risbud MV. Chitosan: a versatile biopolymer for orthopaedic tissue-engineering. Biomaterials. 2005;26(30): 5983-5990.

41. Zhang Y, Zhang M. Synthesis and characterization of macroporous chitosan/calcium phosphate composite scaffolds for tissue engineering. J Biomed Mater Res. 2001;55(3):304-312.

42. Hao W, Hu YY, Wei YY, et al. Collagen I gel can facilitate homogenous bone formation of adipose-derived stem cells in PLGA-beta-TCP scaffold. Cells Tissues Organs. 2008;187(2):89-102.

43. $\mathrm{Xu} \mathrm{HH}$, Simon CG. Fast setting calcium phosphate-chitosan scaffold: mechanical properties and biocompatibility. Biomaterials. 2005;26(12): 1337-1348.

44. Cerruti M, Sahai N. Silicate biomaterials for orthopaedic and dental implants. Reviews in Mineralogy and Geochemistry. 2006;64(1): 283-313.

45. Ramaswamy Y, Wu C, Zhou H, Zreiqat H. Biological response of human bone cells to zinc-modified Ca-Si-based ceramics. Acta Biomater. 2008;4(5):1487-1497.

46. Zhao Y, Ning C, Chang J. Sol-gel synthesis of Na 2 CaSiO 4 and its in vitro biological behaviors. Journal of Sol-gel Science and Technology. 2009;52(1):69-74.

47. Chen M, Le DQ, Baatrup A, et al. Self-assembled composite matrix in a hierarchical 3-D scaffold for bone tissue engineering. Acta Biomater. 2011;7(5):2244-2255.

48. Benson LV. A tabulation and evaluation of ion exchange data on smectites. Environ Geol. 1982;4(1):23-29.

49. Lima IS, Airoldi C. A thermodynamic investigation on chitosan-divalent cation interactions. Thermochim Acta. 2004;421(1-2):133-139.

50. NV M, Kumar R. A review of chitin and chitosan applications. React Funct Polym. 2000;46(1):1-27.

51. Rinaudo M. Chitin and chitosan: properties and applications. Prog Polym Sci. 2006;31(7):603-632.

52. Koide S. Chitin-chitosan: properties, benefits and risks. Nutr Res. 1998;18(6):1091-1101.

53. Seol YJ, Lee JY, Park YJ, et al. Chitosan sponges as tissue engineering scaffolds for bone formation. Biotechnol Lett. 2004;26(13): 1037-1041.

54. Lee YM, Park YJ, Lee SJ, et al. Tissue engineered bone formation using chitosan/tricalcium phosphate sponges. J Periodontol. 2000;71(3): 410-417.

55. Ho MH, Wang DM, Hsieh HJ, et al. Preparation and characterization of RGD-immobilized chitosan scaffolds. Biomaterials. 2005;26(16): 3197-3206.

56. Li Z, Ramay HR, Hauch KD, Xiao D, Zhang M. Chitosan-alginate hybrid scaffolds for bone tissue engineering. Biomaterials. 2005;26(18): 3919-3928.

57. Ma L, Gao C, Mao Z, et al. Collagen/chitosan porous scaffolds with improved biostability for skin tissue engineering. Biomaterials. $2003 ; 24(26): 4833-4841$. 
58. Hein S, Wang K, Stevens WF, Kjems J. Chitosan composites for biomedical applications: status, challenges and perspectives. Materials Science and Technology. 2008;24(9):1053-1061.

59. Mao HQ, Roy K, Troung-Le VL, et al. Chitosan-DNA nanoparticles as gene carriers: synthesis, characterization and transfection efficiency. J Control Release. 2001;70(3):399-421.
60. Greaves M, Wilson M. The adsorption of nucleic acids by montmorillonite. Soil Biol Biochem. 1969;1(4):317-323.

International Journal of Nanomedicine

\section{Publish your work in this journal}

The International Journal of Nanomedicine is an international, peerreviewed journal focusing on the application of nanotechnology in diagnostics, therapeutics, and drug delivery systems throughout the biomedical field. This journal is indexed on PubMed Central, MedLine, CAS, SciSearch $®$, Current Contents $® /$ Clinical Medicine,
Journal Citation Reports/Science Edition, EMBase, Scopus and the Elsevier Bibliographic databases. The manuscript management system is completely online and includes a very quick and fair peer-review system, which is all easy to use. Visit http://www.dovepress.com/ testimonials.php to read real quotes from published authors.

Submit your manuscript here: http://www.dovepress.com/international-journal-of-nanomedicine-journal 